\title{
Climate Change Impacts on Human Health in Bihar
}

\author{
Ritu Sharma \\ School of Ecology and Environment Study, Nalanda University, Rajgir, Bihar
}

Email address:

ritubhardwaj003@gmail.com

\section{To cite this article:}

Ritu Sharma. Climate Change Impacts on Human Health in Bihar. Frontiers in Environmental Microbiology. Vol. 6, No. 3, 2020 , pp. 35-39. doi: $10.11648 /$ j.fem.20200603.12

Received: July 19, 2019; Accepted: April 8, 2020; Published: August 4, 2020

\begin{abstract}
Climate change has become the serious and most concerned topic in the world. It has adverse impacts on physical, socio-economic environment and human health. The objective of this report is to find out the climate change patterns and scrutinize the vulnerability of human's health to the climate change. This study includes the literature reviews and comparative studies of different countries. The findings of this study reveal the direct, indirect and psychological condition impacts of climate change. Due to climate change, the temperature is rising, deviation in seasons are ensuing, summers are extending, winters are getting shorten and rainfall has become irregular; hence vulnerability of human to climate change impacts is increasing day by day. People are becoming more vulnerable to calamities and temperature variability associated diseases such as heat stroke, heat extortion and viral fever by the virtue of vector and water-borne disease such as malaria, diarrhea, cholera, dengue etc. Women and children are more vulnerable to climate change due to lack of adaptation to temperature variability. Surprisingly, Apathy behavior of people towards climate change is reforming Bihar's drought-prone areas into flood-prone and flood-prone areas into drought-prone. Public awareness towards health impacts of climate change could be a good initiative to reduce the vulnerability of women and children.
\end{abstract}

Keywords: Climate Change, Health, Vulnerability, Bihar, Impacts

\section{Introduction}

Climate change is one of the most serious global apprehension of the $21^{\text {st }}$ century. Global sea level has risen about 8 inches in last decade, earth's surface temperature has risen up to $2^{\circ} \mathrm{F}$ (Fahrenheit) since 19 century, glaciers are melting. All these are the evidences of climate change. It is putting the burden on environmental and health determining factors such as air pollution, water quality and accessibility and food security. GHGs emission, exploitation of fossil fuels, pollution and decrease in tree cover are some accountable factors for the change in climate. Climate change has both direct and indirect effects on human health, which include intense heat waves, cold waves, floods, droughts, storms and cyclones are some direct effects, whereas increase in vector and water-borne disease's such as malaria, dengue, cholera, diarrheal incidences and mental ill-being are the indirect impacts of climate change. Women and children are more vulnerable to climate change impacts due to weak immunity. Similarly, developed countries such as India, Bangladesh are more vulnerable to adverse impacts of climate change impacts. World Health Organization predicted that the rise in temperature and irregular rainfall patterns due to anthropogenic climate change of the past 30 years claimed over 150,000 lives annually [2]. It is also determined that climate change causes 2.4 percentage of all cases of diarrhea worldwide and 2 percent of all cases of malaria [6]. Some researchers also revealed that by 2080, approximately 6 billion people of the world would be at a risk of dengue fever as a result of climate change. Poor people are vulnerable to climate change impacts because their drinking water supply and sanitation coverage and infrastructure is also relatively low, which leads to frequent outbreaks of water- borne disease. This is further compounded by frequent floods, heat waves, cold waves that the people experiences. Shortage of skilled health personnel to provide timely and quality health services is another reason behind their vulnerability. Furthermore, geographically people living in coastal regions, hilly areas, water- logged areas, all are vulnerable in different ways. In areas where there is lack of access to clean water supply and sanitation, along with poor hygiene, the burden of diarrheal diseases is also 
increasing. Likewise, When infectious diseases appear in new locations, where people are not aware of its effects and do not have immunity and adequate health facilities, the consequences can be dramatic.

\section{Background}

In India, Bihar is declared as the dirtiest and fifth polluted state of India, in which Patna is the most polluted city. South Bihar is known for drought havoc, while North Bihar is prone in flood debacle. Bihar has an oppressive burden of diseases which arise from natural calamities, poor environmental conditions such as water and vector-borne diseases, poor nutrition status and acute penury in which a day's wage is a significant incentive to skip treatment. These calamities are the evidence of the climate change in Bihar. Bihar's population is mired in penury, they are not able to access their basic amenities. Thus people are more vulnerable to climate change adverse impacts. According to WHO urban air quality database report revealed that after Delhi, Patna has the highest level of airborne particulate matter, PM2.5 considered most harmful to health. Patna with 149 micrograms is the second, next to Delhi. Conferring to a WHO report these figures are 6 times what it considers a 'safe' limit, which is 25 micrograms [19].

\section{Literature Review}

\subsection{Bangladesh Sub Hilly Region Survey}

The author used primary (questionnaire) and secondary data to investigate the vulnerability of women's health to climate change in Kamalganj Upazilla, a sub hilly region of Bangladesh. They studied annually temperature variabilities, rainfall irregularities, diseases profile and affected a number of patients. The study's results revealed that women are more susceptible to climate change impacts by the virtue of unhygienic sanitation, use of contaminated water, traditional cooking system, collecting water from far distances in hot and rainy seasons with bare feet are the reasons behind their increasing intensity of health hazards. Furthermore, lack of nutritious food, healthcare facilities and women's laxity to their health lead to malnutrition and weakness. Moreover, cultural barriers also play a crucial role in the vulnerability of women to heal hazards. Early marriage, high birth rate, illiteracy, lack of awareness, gender discrimination are putting an additional burden of diseases and act as a catalyst to their vulnerability to climate change [14].

\subsection{WHO Regional Strategy}

In WHO 2012 Regional strategy for protecting health from climate change report highlighted the every impact of climate change on health. This report scrutiny defines, how natural disasters cause diseases. Flood will damage the crops and infrastructure, which leads to malnutrition and penury. Similarly, drought and heat waves are also leading cause behind respiratory disease and irritable bowel syndrome. The aftershocks of these calamities come up as migration and psychological stress to exposed people. According to WHO, diarrhoea is responsible for the loss of 73 million DALYs, acute respiratory tract infections for 95 million DALYs, malnutrition for 39 million DALYs and neglected tropical diseases for 19 million DALYs, all of which are directly or indirectly related to sanitation. Worldwide, diarrhoea, acute respiratory infections, malaria and immunizable diseases account for $70 \%$ of the deaths among children aged $0-4$ years [15]. Furthermore, globally at least 3 million children below the age of 5 years die each year because of environment related diseases such as diarrhoeal and acute respiratory diseases. In South-East Asian cities, air pollution causes 530,000 premature deaths per year from respiratory problems [18]. Life expectancy is shortened day by day due to longterm exposure to vehicular pollution and there has been an upturn in incidences of asthma, allergic rhinitis, bronchitis etc. due to the exposure to increased air pollution. By the virtue of climate change, acidification, soil pollution is increasing, which induce people to use the high amount of pesticides and insecticides to increase the production in yield and preclude an upsurge of insects and pests. As a result of extensive use of these pesticides has increased the incidences of premature delivery, low birth weight babies, birth defects, anemia, renal disease and gynecological malignancies [18]. To tackle these issues, WHO initiated some activities that include Increasing awareness of health impacts of climate change among health practitioner, general people, governmental and non-governmental sectors, political, financial and community leaders. Furthermore, strengthening health infrastructures and their system's capacity to provide healthcare facilities to climate-related risks. And the introduction of climate change and its impacts on health into the educational curriculum [19].

\subsection{Temperature Variability and Its Effects on Pathogens}

The author and others in this research paper focus on temperature variability and its effects on pathogens in different countries according to their topography and environmental conditions. They highlighted the temperature at which pathogens are capable to transmit diseases. For many diseases $14-18{ }^{\circ} \mathrm{C}$ is the lower limit and $35-45^{\circ} \mathrm{C}$ temperature is the upper limit for the transmission [17]. Due to climate change, the temperature is rising, in a high temperature, the malarial parasite and other viruses complete their incubation period in female mosquitoes quickly as compared to normal temperature. The author discussed the suitable weather and places, where these vectors can easily survive and transmit diseases. Malaria transmission is more intense at higher altitudes such as East African highlands, due to lack of immunity power [10]. Similarly, Western Europe climate is more appropriate for vivax malaria [1]. Few researches in Kenya reveals that aberration in climate variability accounts up to $26 \%$ of the anomalies in hospitalsbased highland malaria cases [7]. 


\subsection{Direct and Indirect Health Impacts Due to Climate Change}

The author and others focused on primary environmental and social impacts, direct health impacts and indirect health impacts due to climate change. Indirect health impacts, they included injury, death, post-event traumatic stress and diseases risk by the virtue of the climate variabilities such as extreme weather events and heat waves etc. In indirect health impacts, they indicated malnutrition, hygiene and sanitation issues, community and family morale; mental health problems, infectious diseases risks and post-event depression. Furthermore, the author also highlighted the role of the health sector in climate change mitigation and adaptation through the implementation of planned strategies such as to limit the carbon footprint related to the health care system, the designing of a building, transports services and other services should be energy efficient. Similarly, provide adequate health care facilities, which can handle increased patient volume resulting from extreme weather events and ensure adequate stocks of vaccines. There must be some polices for creating green spaces in cities to promote physical and mental health. In the last, they concluded that for a sustainable and healthy lifestyle, there must be a collaboration of health sector with other sectors in reframing how human societies plan, produce, consume, share and generate energy [11].

\subsection{Diseases Caused by Climate Change}

The author illustrate the methods through which we can estimate the diseases caused by climate change. To estimate the burden of diseases attributable to a risk factor, three factors are necessary to evaluate i.e. i) burden of specific disease. ii) Estimation of increase in risk of each diseases per unit increase in exposure. iii) Current population distribution of exposures, or future distribution as estimated by modelling exposure scenarios. They also discussed different scenario, which reveal changes in global climate conditions. Moreover, the author also analyzed the health outcomes associated with the climate change such as direct impacts of heat and cold could cause cardiovascular disease death, food and waterborne diseases could come up as diarrhoeal episodes, vectorborne diseases lead to malaria, dengue cases and natural calamities can cause fatal unintentional injuries and malnutrition [2]. They used different analysis to investigate the correlation of climate variabilities and outbreaks of the diseases. The author used time series analysis to correlate temperature, humidity and rainfall variations to daily hospital admissions in a pediatric diarrhoeal diseases clinic in Lima, Peru. The study came up with a result that $8 \%$ increase in hospital admission per $1{ }^{\circ} \mathrm{C}$ increase in temperature through the whole year [3]. Similarly, the author performed the time series analysis to correlate temperature and rainfall to monthly reported diarrhoeal incidences in Fiji. The study revealed that there is $3 \%$ increase in diarrhoeal incidences as per $1{ }^{\circ} \mathrm{C}$ temperature rises [16]. These studies exhibit clear evidence of increase in diarrhoeal incidences with increase in temperature. The author claimed that, "This is a web of interactions between ecosystems, climate and human societies, which influences the occurrence of infections" [8].

\subsection{Methods to Access the Effects of Climate Change}

The author discussed the various methods to access the effects of climate change on human health, which include spatial analogue studies, predictive modelling, biological models or empirical-statistical models and expert judgement. For scrutiny require current environmental stresses and issues. After this analogue study consist of qualitative and quantitative methods. Furthermore, empirical study include analysis of relationships between trends in climate and indicators or health status for example: increase in malaria incidences and climate, heat attributable mortality. Three types of analogue studies are mentioned i.e. historical trends (e.g. local warming trend, decrease in precipitation), historical events (e.g. extreme weather events, floods, drought) and cross-sectional spatial analogues (e.g. comparative study of one location to another location). Assessment also consist of study area, in which climate characteristics such as temperature and rainfall, disease profile of study area and assessment of correlation between climate variability and diseases should be considered [12].

\subsection{Pacific Island Vulnerable to Climate Change}

The author states that in 2001, the IPCC declared that Pacific island is the nation's most vulnerable island to the climate change [9]. Furthermore, they also identified the factors those are responsible for the shortage of skilled health workers, which include a lack of effective planning, limited health budgets, migration of health workers, limited employment opportunities, low salaries and poor working conditions, weak support and supervision, health workers shortage especially in the rural and remote areas and limited opportunities for professional development. Moreover, the author also evaluate the direct and indirect health effects due to climate change. Few direct health effects are shortage of food and water, water and food-borne diseases, vector-borne and rodent-borne diseases, psychological and nutritional disorders, air pollution-related health effects, temperaturerelated illness and deaths and extreme weather-related health effects. Indirect effects are microbial contamination pathways, vector-borne diseases transmission, socioeconomics demographics, infrastructure damage and migration [13]. These natural calamities are due to climate change, which shattered the lives of people completely along with cause many health impacts such as some post-flood health impacts are death and direct injuries, including bites from animals, insect and rodent-borne diseases including malaria, dengue, leptospirosis and yellow fever. Water-borne disease including cholera, schistosomiasis and cryptosporidium, food-borne diseases include diarrhoeal diseases, cholera, food poisoning, typhoid and hepatitis A \& E [4]. Respiratory diseases include asthma, bronchitis and respiratory allergies, heat-related diseases include sunstroke, sunburn, heat stress, heat exhaustion and dehydration. 
Dermatitis, malnutrition, migration, anxiety, stress and schizophrenia [5]. To tackle these problems the author explained the gaps and adaptation measures.

\section{Results}

This study reveals the relationship between climate change and human's health together with the spatial patterns of the vulnerability to diseases in the context of climate changing. By the virtue of increase in temperature cause upturn in the mortality rate of heat stroke and heat waves, which leads to cardiovascular and respiratory diseases. Floods, landslides and windstorms disrupt water supply and sanitation, it also provides breeding sites for mosquitoes and causes posttraumatic stress disorders, which cause infectious diseases and mental disorders. The temperature and rainfall variability affect water availability, quality and also disseminate water and vector-borne diseases. This is a web of interactions between ecosystems, climate and human societies, which influences the occurrence of infections [8]. Therefore, to negotiate these issues the key demand is health care system collaboration with other systems and climate change adaptation programmers should be undertaken by governmental and non-governmental organizations. Furthermore, public awareness towards health impacts of climate change could be a good initiative to reduce the vulnerability of women and children. Nonetheless, the addition of climate change projections in the health policies and commencement of climate change impacts on human health in educational curriculum deserves a careful consideration.

\section{Conclusion}

For the evaluation of climate change impacts on human health, a proper scrutiny of data is required. Many countries and states are analyzing data and information to take appropriate measures to prevent these effects. As of now, the state Bihar has not undertaken any such vulnerability assessment of human health to climate change impacts. Bihar is an epitome of pollution and penury. Apathy behavior of people towards climate change is reforming Bihar's droughtprone areas into flood-prone and flood-prone areas into drought-prone. Simultaneously, the rate of debacles is also upturning day by day, which result into increase in the mortality and morbidity rates. To overcome and evaluate the situation properly, an adequate assessment needs to be carried out on the basis of secondary as well as primary data to understand the extent of diseases burden that may occur due to climate change and population projections and assessment of development induced risks should also be considered. To bring a climate resilient health system an assessment of a number of additional health centers and skilled health personnel that would be required should be done. Nonetheless, development of a vulnerability and risk framework in terms of health and climate change. With all of the work ahead of us, we need to begin now.

\section{References}

[1] Baldari M et al. Malaria in Maremma, Italy. Lancet, 1988, 351: 1246-1247.

[2] Campbell-Lendrum, D. H., Pruss-Ustun, A., \& Corvalan, C. (2003). How much disease could climate change cause. Climate change and human health: risks and responses. Geneva: WHO, 133-158.

[3] Checkley, W. et al. Effects of El Niño and ambient temperature on hospital admissions for diarrhoeal diseases in Peruvian children. Lancet 355 (9202): 442-450 (2000).

[4] Ebi KL, Lewis ND, Corvalan C. Climate Variability and Change and Their Potential Health Effects in Small Island States: Information for Adaptation Planning in the Health Sector. Geneva. UNEP, WHO, WMO. 2005. http://www.who.int/globalchange/publications/climvariab.pd f.

[5] Few R, Ahern M, Matthies F, Kovats S. Floods, health and climate change: a strategic review. Working Paper 63. Norwich. Tyndall Centre for Climate Change Research. 2004 November.

http://www.eird.org/isdrbiblio/PDF/Floods\%20health\%20and $\% 20$ climate $\% 20$ change.pdf.

[6] Foreign Policy Blog Network. The Aral Sea disaster, part 3: living the climate change prophecy. Available at: $\mathrm{http}: / /$ centralasia.foreignpolicyblogs.com/2007/04/11/the-aralsea-disaster-part-3-living-the-climate-changeprophecy/ (accessed on 20 June 2017).

[7] Githeko, A. K., Lindsay, S. W., Confalonieri, U. E., \& Patz, J. A. (2000). Climate change and vector-borne diseases: a regional analysis. Bulletin of the World Health Organization, 78 (9), 1136-1147.

[8] Hales, S., Edwards, S. J., \& Kovats, R. S. (2003). Impacts on health of climate extremes. Climate change and health: risks and responses. Geneva, World Health Organization.

[9] IPCC. Climate change 2001: Impacts, adaptation and vulnerability. Cambridge. 2001.

[10] Lindsay SW, Martens WJ. Malaria in the African highlands: past, present and future. Bulletin of the World Health Organization, 1998, 76: 33-45.

[11] McMichael, A. J. (2013). Globalization, climate change, and human health. New England Journal of Medicine, 368 (14), 1335-1343.

[12] McMichael, A. J., Haines, A., \& Kovats, R. S. (2001). Methods to assess the effects of climate change on health. Health effects of climate change in the UK.

[13] McMichael AJ, Woodruff RE, Hales S. Climate change and human health: present and future risks. The Lancet. 2006; 367 (9513): 859-869.

[14] Naim, J., \& Paul, S. K. vulnerability of women's health to climate change impacts: a study on sub-hilly region of Bangladesh.

[15] Pelletier DL et al. The effects of malnutrition on child mortality in developing countries. Bulletin of the World Health Organization, 1995, 73 (4): 443-448. 
[16] Singh, R. B. K. et al. The influence of climate variation and change on diarrhoeal disease in the Pacific Islands. Environmental Health Perspectives 109 (2): 155-159 (2001).

[17] Watts DM et al. Effect of temperature on the vector efficiency of Aedes aegypti for dengue to virus. American Journal of Tropical Medicine and Hygiene, 1987, 36: 143-152.
[18] WHO. Research to assess impact of climate change on communicable diseases. Report of an informal consultation. New Delhi, WHO SEARO, 2010 [SEACD-223].

[19] WHO SEARO. Regional action plan to protect human health from climate change. New Delhi, WHO SEARO, December 2007. 